\title{
Téoros
}

Revue de recherche en tourisme

\section{Classification des établissements hôteliers}

\section{François de Grandpré}

Volume 8, numéro 2, juillet 1989

Hébergement et tourisme

URI : https://id.erudit.org/iderudit/1080319ar

DOI : https://doi.org/10.7202/1080319ar

Aller au sommaire du numéro

Éditeur(s)

Université du Québec à Montréal

ISSN

0712-8657 (imprimé)

1923-2705 (numérique)

Découvrir la revue

Citer cet article

de Grandpré, F. (1989). Classification des établissements hôteliers. Téoros, 8(2), 10-12. https://doi.org/10.7202/1080319ar d'utilisation que vous pouvez consulter en ligne.

https://apropos.erudit.org/fr/usagers/politique-dutilisation/ 


\section{Classification des établis- sements hôteliers}

En 1984-1985, une équipe de chercheurs de I'Universitć du Québec à Trois-Rivières" procédait à la classification des pourvoiries de chasse et de pểche du Québec. A l'époque, l'élaboration de la recherche avait témoigné de la rareté des documents de référence sur les systèmes de classification et ainsi provoquer une étude des systèmes de classification des équipements touristiques Centré essentiellement sur la classification hôtelière, le présent article s"intéresse à deux éléments de cette étude: les caractéristiques des guides hôteliers et les processus de classification.

Excellentes sources d'information pour le touriste et outils de développement touristique indéniables, il semble que les guides de classification hồlelière, comme Michelin en France ou celui de l'American Automobile Association aux États-Unis, se multiplient à travers le monde sans règle ni standard depuis près d'un siècle. En conséquence, malgré leur apparente similitude, les guides proposes aux voyageurs ainsi que les systemes de classification utilisés different considérablement de l'un à l"autre.

Partant de ce fait et dans l'intention de contribuer à une meilleure connaissance des pratiques de classification hôtelière, nous ferons état dans un premier temps des caractéristiques des guides hoteliers et ensuite, du processus de classification.

\section{Principales caractéristiques des guides}

Les guides de classification hôtelière possèdent tous leurs particularités. En effet, le format, la présentation, les symboles utilisés, la clientèle cible, l'objet et même le service sur lequel porte la classification font de chacun d'eux un outil particulier. Le tableau 1 fait ressortir quelques caractéristiques de vingt-huit guides de classification hôtelière en usage dans seize pays' Certaines données de l'analyse proviennent des études de Macken (1973), Chapman' (1980) et Vine (1981) et sont utilisées comme complément d'information.

L'année de publication est un elément à considérer dans l'analyse des guides de classification, les informations et leur format pouvant varier d'une édition à l'autre. Au tableau 1, la plupart des guides analysés datent de 1986. La mention projet signifie que le guide est ou a déjà étê en cours de développement comme c'est le cas au Canada pour la classification des êtablissements hôteliers des provinces de l'Atlantique. Soulignons que le projet de classification des établissements de la Nouvelle-Écosse, développé au début des années '80 parr le Towrism Industry Association of Nova Scotia, acété mis de côté au profit du projet du Economic Planning Group of Canada qui regroupe les établissements hôteliers des quatre provinces de l'Atlantique (Nouveau-Brunswick, Nouvelle-Écosse, Ile-du-Prince-Édouard et Terre-Neuve), D'autre part, toujours en ce qui concerne le Canada. l'étude de Macke indiquait, voilà 16 ans, l'existence dune classification des établissements hôteliers au Manitoba et en Saskatchewan. Nous ne savons pas si ces guides existent toujours, raison pour laquelle ils n'apparaissent pas dans le tableau.

Le mode d'entréce des établissements dans le guide est aussi un élément susceptible de faire varier les informations d'un guide à l'autre. En général, il semble qu'elle s'effectue selon trois modes: laclassification choisie, la classification volontaire ou encore la classification obligatoire.

La classification choisie se caractérise par le fait que l'organisme classificateur procède à une sélection desétablissements à partir d'exigences précises. Cette méthode produit des guides qui ne contiennent que les meilleurs établissements. C'est le cas pour les guides français tels "Le Bottin Gourmand", Gault Millau, les guides Hachette et Michelin ainsi que pour les guides américains AAA, Brewster, Exxon, Fisher et Mobil.

Par ailleurs, une classificationest dite volontaire lorsque l'opérateur fait lui-même les démarches nécessaires pour que son établissement soit classifié dans un guide. C'est la méthode utilisée par le gouvernement ontarien et éventuellement par les provinces maritimes. Mis à part ces provinces canadiennes, il semble que l'Angleterre soit le seul pays à proceder à une classification volontaire,

Quant à la classification obligatoire, elle inclut la totalité des établissements situés sur le territoire concerne. Seuls les organismes gouvernementaux ou mandatés par ces demiers peuvent se permettre cette approche, Le Québec, la Colombie, I'Espagne, le Ghana, I'Irlande du Nord et le Sri Lanka sont des pays qui procèdent à une classification obligatoire.
* Monsieur Francois de Grandpré est assistant de recherche au Département des Sciences du loisir à I'Université du Québec à Trois-Rivières. 
TABLEAU 1

Quelques caractéristiques des guides hôteliers

\begin{tabular}{|c|c|c|c|c|c|c|}
\hline Pays & $\begin{array}{l}\text { Année de la } \\
\text { publication" }\end{array}$ & $\begin{array}{l}\text { Choisie } \\
\text { wolon - } \\
\text { taire } \\
\text { whliga - } \\
\text { toire }\end{array}$ & $\begin{array}{l}\text { Club au - } \\
\text { tomobile } \\
\text { Gouwer - } \\
\text { nement } \\
\text { Firme } \\
\text { neutre }\end{array}$ & $\begin{array}{l}\text { Nombre } \\
\text { de } \\
\text { classes }\end{array}$ & $\begin{array}{l}\text { Nombre } \\
\text { de cate - } \\
\text { gories }\end{array}$ & $\begin{array}{l}\text { Inclut } \\
\text { restau - } \\
\text { rants } \\
\text { oui/non }\end{array}$ \\
\hline $\begin{array}{l}\text { CANADA } \\
\text { N.-Eoosse } \\
\text { (TIANS) }\end{array}$ & PROJET & v & G & 5 & 7 & N \\
\hline Ontario & 1986 & V & $\mathrm{G}$ & 5 & 9 & $\mathbb{N}$ \\
\hline $\begin{array}{l}\text { P. Atlantique } \\
\text { (EPC) }\end{array}$ & PROJET & v & $\mathrm{G}$ & 5 & ND & N \\
\hline Québee & 1986 & 0 & $\mathrm{G}$ & 6 & 5 & $\mathrm{~N}$ \\
\hline ETATS-UNIS & & & & & & \\
\hline AAA & 1986 & C & C & $5^{* * 4}$ & 13 & 0 \\
\hline Brewster & 1984 & C & $\mathrm{F}$ & 4 & ND & 0 \\
\hline Exxon & 1981 & $\mathrm{C}$ & $\mathrm{F}$ & 5 & ND & 0 \\
\hline Fisher & 1986 & C & $\mathrm{F}$ & $S^{* * *}$ & 1 & 0 \\
\hline Mobil & 1987 & $\mathrm{C}$ & $\mathrm{F}$ & 5 & 6 & 0 \\
\hline $\begin{array}{l}\text { AFRIQUE DU } \\
\text { SUD }\end{array}$ & 1985 & ND & G & 5 & 5 & $\mathrm{~N}$ \\
\hline ALLEMAGNE & ND & $\mathrm{ND}$ & $\mathrm{G}$ & 6 & ND & ND \\
\hline ANGLETERE & & & & & & \\
\hline $\mathrm{AA}$ & 1986 & $\mathrm{v}$ & C & 5 & 1 & 0 \\
\hline English T. Board & 1980 & $\mathrm{~V}$ & $\mathrm{G}$ & 6 & ND & ND \\
\hline Royal A. Club & $\mathrm{ND}$ & $\mathrm{v}$ & $\mathrm{C}$ & ND & ND & ND \\
\hline AUSTRALIE & 1986 & ND & C & 9 & 2 & $\mathrm{~N}$ \\
\hline AUTRICHE & 8687 & ND & C & 5 & 7 & $\mathrm{~N}$ \\
\hline COLOMBIE & $\mathrm{ND}$ & 0 & $G$ & 5 & 4 & ND \\
\hline ESPAGNE & $\mathrm{ND}$ & 0 & $G$ & 5 & 4 & $\mathrm{~N}$ \\
\hline $\begin{array}{l}\text { FRANCE } \\
\text { le Bottin }\end{array}$ & & & & & & \\
\hline Gourmand & 1986 & $\mathrm{C}$ & F & 5 & 1 & 0 \\
\hline Gault Millau & 1986 & C & F & 5 & 1 & 0 \\
\hline Hachette & 1986 & $\mathrm{C}$ & $\mathrm{F}$ & 4 & 1 & 0 \\
\hline Michelin & 1986 & $\mathrm{C}$ & $\mathrm{F}$ & 5 & 1 & 0 \\
\hline GHANA & PROJET & 0 & $\mathrm{G}$ & 5 & ND & ND \\
\hline HOLLANDE & ND & ND & $\mathrm{G}$ & ND & $\mathrm{ND}$ & $\mathrm{ND}$ \\
\hline INDE & ND & ND & ND & 5 & ND & ND \\
\hline $\begin{array}{l}\text { IRLANDE } \\
\text { IRLANDE DU }\end{array}$ & ND & ND & $\mathrm{G}$ & 5 & 4 & $\mathrm{ND}$ \\
\hline NORD & 86187 & 0 & G & $5 * *$ & 2 & $\mathrm{~N}$ \\
\hline SRI LANKA & $\mathrm{ND}$ & 0 & $G$ & 5 & $\mathrm{ND}$ & ND \\
\hline
\end{tabular}

Legende:

ND: Information non disponible.

*: Annoser de la publication des guides recensis.

*k: Sans compler une classe audditionintle qui n'utilise ancun symbole.
Qui classifie? L'ensemble des organismes classificateurs est constitué de trois institutions dislinctes; les clubs automobiles, les gouvernements et les firmes neutres:, Dans notre analyse. sur l'ensemble des guides recensés, $52 \%$ des classifications ont ête effectuées par un gouvernement, $29 \%$ par une firme neutre et $19 \%$ par un club automobilc. Il est intéressant de noter à ce propos qu'au Canada, la classification hótelière est toujours de source gouvernementale et que, dans cecréneau d'activité, seuls les Ëtats-Unis et la France ont une demande suffisante pour permettre à des firmes neuires ou privês d'être actives.

Nous faisons une distinetion entre les classes et les catégories utilisées dans les guides répertorics. Une classe réfere à une mesure de qualité, par exemple "hotel de confort superieur" el une catégorie réfere soit à une mesure de quanlite d'equipement, "hơtel bien équipéc" ou à la mature de l'établissement "hötel, motel, ete.".

Lat quatrieme colone du tableau indique le nombre de classes utilisbes dans chaque guide. Ce nombre varie entre quatre et neut, cing ctant le nombre de classes le plus souvent utilisées. Vingt des vingt-six $(77 \%)$ des guides pour lesquels nous détenons cette information ont un système à cinq classes, trois $(11 \%)$ utilisent six classes, deux $(8 \%)$ en utilisent deux et un $(4 \%)$ en utilise neuf. Des vingt systèmes à cinq classes, trois en incluent une sixiême sans symbole. Dans le cas d'une classification choisie, cette classe sans symbole représente des établissements d'un certain mérite mais qui n'atteignent pas le standard pour se mériter un symbole. Dans le cas d'une classification obligatoire, nawoir aucun symbole signifie que l'établissement ne rencontre pas les normes minimales.

L'avant dernière culonne indique le nombre de categories utilisees dans les guides. Nous les avons divisées en trois groupes. D'abond celui qui classifie indifferemment tous les types d'établissements sous le terme générique "hởtel" $(33 \%)$, ensuite celui qui utilise deux catégoriegen (II ro et finalement celui qui utilise quatre catégories et plus $(56 \%)$.

Enfin, la dernière colonne indique si les guides incluent les restaurants autres que ceux relies aux établissements hôteliers, On peut voir que dix $(53 \%)$ des dix-neuf guides les incluent.

\section{Processus de classification}

Létude des guides et des systèmes a indiqué des grandes tendances dans les processus de classification dont les principaux éléments sont schématises à la figure 1. II importe de preciser que le schéma est une représentation sommaire des procedes les plus fréquemment rencontrés et non une description complète de ceux-ci.

La figure montre que selon l'organisme qui classifie, on oblient une classificaton fonda- 
mentalement differente. Les trois principaux organismes classificateurs sont, dans l'ordre, les gouvernements, les firmes neutres et les clubs automobiles, 11 arrive aussi parfois que des firmes non-neutres (habituellement représentées par l'association des hôteliers) participent au developpement de systèmes de classification.

Comme le but poursuivi est différent pour chacun des organismes classificateurs, les moyens qu'ils prennent pour classifier varient. La figure 1 illustre comment le but vise inthence la condition dentré et la technique de collecte utilisées. Les deux premiers organismes classificateurs, le gouvernement et la firme non-neutre, visent à augmenter la qualité de l'industrie et à développer cette demière. Les deux organismes suivent le même cheminement à partir dece point, visant tous deux une meilleure mise en marché du produit. Le gouvernement focalise davantage sur le futur de l'industrie en espérant que les standards de classification feront augmenter la qualité et la firme non-neutre souhaite que le systeme soit un outil de marketing pour l'ensemble des operateurs en tant qu'industric et pour chacun d'eux en tant que propriétaires. Pour atteindre leur but, et surtout pour augnenter la qualité de l'industrie, les organis. mes classificateurs doivent s'assurer la plus grande participation possible. En fait, les organismes classificateurs qui representent un gou= vernement rendent souvent la classification obligatoire. Ceci s'applique à tous les systemes de classifications observés et en opération. Seul le gouvemement ontarien fait exception puisqu'il classifie sur une base volontaire. Dans ce dernier cas, tous les hötels se trouvent dans le guide, mais seuls les établissements dont les propriétaires ont fait les démarches nécessaires sont classifiés. Les gouvernements possèdent

\section{Figure 1}

Tendances dominantes dans les processus de classification utilisés

\begin{tabular}{|c|c|c|c|c|}
\hline $\begin{array}{l}\text { Organisme } \\
\text { classificateur }\end{array}$ & Gouvernemen & $\begin{array}{l}\text { Fime } \\
\text { non-neutre }\end{array}$ & Club automobile & $\begin{array}{l}\text { Firme: } \\
\text { neutre }\end{array}$ \\
\hline But & $\begin{array}{l}\text { Augmenter la } \\
\text { qualité }\end{array}$ & $\begin{array}{l}\text { Développer } \\
\text { l'industrie }\end{array}$ & $\begin{array}{l}\text { Offrir un service } \\
\text { aux membres }\end{array}$ & $\begin{array}{l}\text { Faire des } \\
\text { profits }\end{array}$ \\
\hline Conditions & $\begin{array}{l}\text { Obligatoire: } \\
\text { inclut tous } \\
\text { les ctablissements }\end{array}$ & $\begin{array}{l}\text { Choisie: selection } \\
\text { d'établissement }\end{array}$ & $\begin{array}{l}\text { Volontaire" : } \\
\text { selon la demande } \\
\text { des operateurs }\end{array}$ & \\
\hline $\begin{array}{l}\text { Technique } \\
\text { de collecte* }\end{array}$ & $\begin{array}{l}\text { Questionnaire } \\
\text { aux operateurs }\end{array}$ & $\begin{array}{l}\text { Classificateur } \\
\text { sans expertise } \\
\text { du domaine }\end{array}$ & $\begin{array}{l}\text { Classificateur } \\
\text { expert du } \\
\text { domaine }\end{array}$ & $\begin{array}{l}\text { Sondage } \\
\text { aupres de } \\
\text { la clientèle }\end{array}$ \\
\hline $\begin{array}{l}\text { Type de } \\
\text { classification }\end{array}$ & Niveau de service & Qualiué des lieux & Qualité des services & \\
\hline $\begin{array}{l}\text { Le pydime of } \\
\text { mitue sil ks }\end{array}$ & 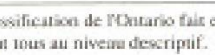 & 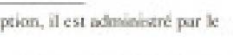 & geuvertancat mais ne slassify & 2 pas tous les atablissment: \\
\hline
\end{tabular}

aliments, etc.. Enfin, la plus difficile à réaliser, mais aussi la plus porteuse d'information, est la classification qui permet de mesurer la "qualité des services". Dans ce type de classification, le classificateur doit déterminer le degré de qual itế des services offerts. Il doit done posséder une expertise lui permettant d'appréhender la réalité et de porter un jugement juste et equitable sur des éléments souvent complexes ả évaluer.

\section{Conclusion}

L'analyse des systemes de classification hôtelière permet de mieux connaâtre et aussi de mieux comprendre les guides et les processus de classification. La mise en relief de differentes variables montre qu"il existe des caractéristiquescommunes entre les différents guides et des grandes tendances au niveau du processus de classification. On constate qu'un des rouges importants du processus de classification est lorganisme classificateur lui-même, son statut et le but quil poursuit.

Il est certain que les limites de cet article oblige à passer sous silenee plusieurs aspects de la classification hôtelière qui sont étudiés dans l'étude sur les systèmes de classification ", étude quià son tour ne comble certainement pas le trou dans la litterature à ce sujet. Aussi serait-il souhaitable que la recherche se poursuive pour, par exemple, préciser l'impact économique et sociales des classifications sur les pays hôtes ou encore mesure l'apport rél des guides de classification pour l'hötelier.

\section{NOTES EXPLICATIVES}

(1) Rocherche mandate par le ministere de la Chase et de la Peche el dingere par Andre Barabe, Mas d'Anours. Gilles Pronowost, Robert Soubrier.

(2) DE GRANDPRE, François (1987), Analyse comparalive des systemes de classification et des auides touristiques en usage dans certains pays oceidentaus.

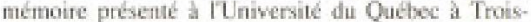
Rivieres, IBR pages.

(3) Certains organismes classifien dautres pays que le beu mais nous ne les avons inclus qu' une seule foss, sous leur pays dionigine.

(4) MACKE. Markene (1973). Report on Accomodation Classification Systems, Canada, septembre, 17 pages.

(5) CHAPMAN, Masa (I940), Comparative Study of Classification Systems, Angleterr. 66 pages.

(6) VINE, P.A.1 (1981), Hon Classiffoulien - An or Sricuce? dans International Journal of Tourism Manigemeat, vol. 2, no 1, mars, pp, 18-29,

(7) The Economic Planning Groum of Canada, Atlantic Provinces Accomodation Grading Systen Study, rappont final, janvier 1984, 250 pages.

(8) C'est-a-dire un organisme priwequi gia aucun lien direst avec l'undustrie hobleliere, qui riest ni un organisme gouvememental ni un club auchnobile.

(9) Les systhmes aे deuk catcgories sont celui du Wariowal Revd awd Morvist Assacianon (Australic) qui utiliac les catégories "Hötel" el "Molel" el célui du Norherio Iratand Tourin Board qui unilise "Hōtel" en "Pension de fanille" ("gueshouse").

(10) DE GRANDPRÉ, Francois, op. cit. 